\title{
Adherence issues related to sublingual immunotherapy as perceived by allergists
}

\author{
This article was published in the following Dove Press journal: \\ Patient Preference and Adherence \\ 14 June 2010 \\ Number of times this article has been viewed
}

\section{Silvia Scurati' \\ Franco Frati' \\ Gianni Passalacqua ${ }^{2}$ \\ Paola Puccinelli' \\ Cecile Hilaire' \\ Cristoforo Incorvaia ${ }^{3}$ \\ Italian Study Group on \\ SLIT Compliance}

'Scientific and Medical Department, Stallergenes, Milan, Italy; ${ }^{2}$ Allergy and Respiratory Diseases, Department of Internal Medicine, Genoa; ${ }^{3}$ Allergy/ Pulmonary Rehabilitation, ICP

Hospital, Milan, Italy
Correspondence: Cristoforo Incorvaia Allergy/Pulmonary Rehabilitation, ICP Hospital, Milan, Italy, Via Bignami I,20 I 26 Milan, Italy

Tel +390257993289

Fax +39025 7993276

Email cristoforo.incorvaia@gmail.com
Objectives: Sublingual immunotherapy (SLIT) is a viable alternative to subcutaneous immunotherapy to treat allergic rhinitis and asthma, and is widely used in clinical practice in many European countries. The clinical efficacy of SLIT has been established in a number of clinical trials and meta-analyses. However, because SLIT is self-administered by patients without medical supervision, the degree of patient adherence with treatment is still a concern. The objective of this study was to evaluate the perception by allergists of issues related to SLIT adherence.

Methods: We performed a questionnaire-based survey of 296 Italian allergists, based on the adherence issues known from previous studies. The perception of importance of each item was assessed by a VAS scale ranging from 0 to 10 .

Results: Patient perception of clinical efficacy was considered the most important factor (ranked 1 by 54\% of allergists), followed by the possibility of reimbursement (ranked 1 by 34\%), and by the absence of side effects (ranked 1 by $21 \%$ ). Patient education, regular follow-up, and ease of use of SLIT were ranked first by less than $20 \%$ of allergists.

Conclusion: These findings indicate that clinical efficacy, cost, and side effects are perceived as the major issues influencing patient adherence to SLIT, and that further improvement of adherence is likely to be achieved by improving the patient information provided by prescribers.

Keywords: adherence, sublingual immunotherapy, efficacy, cost, side effects

\section{Background}

Sublingual immunotherapy (SLIT) is currently accepted as a viable alternative to subcutaneous immunotherapy (SCIT) to treat allergic rhinitis and asthma, ${ }^{1}$ and is widely used in clinical practice in many European countries. The clinical efficacy of SLIT has been established in a number of clinical trials and meta-analyses, ${ }^{2}$ and its safety profile has been reported to be satisfactory in adults and children using high allergen doses. ${ }^{3}$ However, because SLIT is self-administered by patients without medical supervision, assessment of adherence to treatment is still a concern, although the advantage of home administration does remove one of the main causes for no adherence, ie, the need to go to a physician's office for treatment, which was previously the case with SCIT. ${ }^{4}$ The first studies of SCIT in the 1990s reported an adherence rate of about $50 \%$, with better rates (up to $75 \%$ ) subsequently found using less demanding injection schedules. ${ }^{4}$ With SLIT, higher adherence rates of up to $90 \%$ have been described. ${ }^{4}$

Adherence is commonly defined as the extent to which the patient's behavior matches the treatment recommendations from the prescriber ${ }^{5}$ and is essential for the success of any medical treatment, especially for chronic diseases, such as rhinitis and asthma. Medication intake can be assessed using several direct or indirect methods. Direct methods, 
which measure the concentration of a specific drug in blood or urine, cannot be used for assessment of SLIT adherence. Indirect methods require periodic counting of leftover doses or use of questionnaires in which patients report the amount and frequency of drug intake. Considering the recent literature, adherence to SLIT in clinical trials and postmarketing surveys would appear overall to be satisfactory, ranging from $75 \%$ to $90 \%,{ }^{6-8}$ with inconvenience and cost of treatment being the main causes for nonadherence. All these studies focused on patients, but the role of physicians must not be overlooked, because their attitude towards a treatment influences the information they provide to patients when prescribing SLIT in their everyday clinical practice which, in turn, may influence patient adherence. For example, insufficient information on the common side effects of SLIT, such as local reactions in the mouth, may lead to unnecessary treatment modification and even to withdrawal. ${ }^{9}$ The present questionnaire-based survey sought the opinions of Italian allergists on SLIT adherence.

\section{Methods}

A multiple-choice questionnaire was administered to 325 Italian allergy specialists, randomly selected from a national list, who commonly recommend treatment with SLIT. The questionnaire comprised 10 questions concerning different factors which can favorably influence patient adherence with SLIT. These factors were perceived efficacy, tolerability, cost reimbursement, administration regimen (pre-coseasonal/ continuous), ease of administration, regular contact with the patient, approval of the treatment by the general practitioner, patient education, and follow-up visits. The allergist's perception of the proportion of patients completing the recommended SLIT duration of three years was used to estimate the extent of follow-up. ${ }^{1}$ Each question was to be answered using a $10 \mathrm{~cm}$ visual analog scale (VAS) ranging from 1 ("extremely important") to 10 ("of no relevance"). The VAS is recognized as a very useful tool for detecting an individual's perception of a specific medical issue. ${ }^{10}$ The physicians were also asked to choose the most relevant factor that, in their opinion, impaired adherence.

\section{Results}

Of the 325 allergy specialists contacted, 296 (91\%) returned a valid questionnaire with all questions answered. The mean age of the allergists was 51 years; $40.5 \%$ were female, and $5 \%$ were in private practice, $60 \%$ in hospital practice, and $35 \%$ worked in a territorial health care setting.

The patient's perception of clinical efficacy was considered to be the most important factor influencing adherence, and was ranked 1 ("extremely important") by 54\% of allergists; this finding was further confirmed by considering the sum of the first three ranks (Tables 1 and 2), with $78.1 \%$ of allergists considering this to be the most important factor. The second important factor influencing adherence was considered to be the possibility of cost reimbursement by the National Health Service, with 34\% of allergists ranking this first (Table 3). The absence of treatment side effects was considered to be the third important factor influencing adherence, ranked first by $21.3 \%$ of allergists (Table 4 ).

The remaining factors ranked first in less than $20 \%$ of the answers. Patient education, acceptance by the general practitioner, and regular follow-up visits were not considered particularly relevant to maintenance of adherence. Also, the ease of administration of SLIT received low consideration by allergists. Considering the main causes of treatment discontinuation, the cost of SLIT was reported by $98 \%$ of physicians, followed by side effects (48\%), and lack of perceived clinical efficacy (42\%).

When asked to define a method to increase patient adherence, the majority (57.8\%) of allergists chose telephone contact as a more useful tool than frequent follow-up visits. Finally, most allergists $(57.8 \%)$ thought that a three-year course of SLIT would have an adherence of 50\%-80\%, while $29.4 \%$ estimated adherence to be over $80 \%$, and only $7.1 \%$ estimated adherence to be lower than $50 \%$ (Table 5).

\section{Discussion}

Adherence to the recommended therapeutic regimen is crucial in all aspects of medicine, ${ }^{1-13}$ especially in chronic diseases, such as rhinitis and asthma. This is particularly true with SLIT which is self-administered by patients at home and involves prolonged treatment without medical supervision, and the potential therefore exists for arbitrary modification of the dosing regimen, which can lead to a progressive

Table I Percentage of allergists who ranked questionnaire issues influencing adherence to SLIT at I-3

\begin{tabular}{lll}
\hline & $\mathbf{n}$ & $\%$ \\
\hline Perceived efficacy of treatment by patients & 231 & 78.1 \\
Tolerability & 196 & 66.2 \\
Cost reimbursement & 171 & 57.8 \\
Patient education & 117 & 39.4 \\
Ease of use & 106 & 35.7 \\
Regular physician-patient contact & 95 & 32.1 \\
Regular follow-up visits & 90 & 30.4 \\
Administration regimen (pre-co seasonal) & 72 & 24.3 \\
Administration regimen (continuous) & 65 & 21.9 \\
Approval from general practitioner & 49 & 16.6 \\
\hline
\end{tabular}

Abbreviation: SLIT, sublingual immunotherapy. 
Table 2 Allergists' ranking of perception of efficacy as the most important factor in adherence to SLIT

\begin{tabular}{lllll}
\hline Ranking & $\mathbf{n}$ & $\%$ & \multicolumn{2}{l}{ Cl 95\% } \\
\cline { 3 - 5 } & & & Lower & Upper \\
\hline 1 & 161 & 54.4 & 48.7 & 60.1 \\
2 & 49 & 16.6 & 12.3 & 20.8 \\
3 & 21 & 7.1 & 4.2 & 10.0 \\
4 & 7 & 2.4 & 0.6 & 4.1 \\
5 & 15 & 5.1 & 2.6 & 7.6 \\
6 & 5 & 1.7 & 0.2 & 3.2 \\
7 & 7 & 2.4 & 0.6 & 4.1 \\
8 & 13 & 4.4 & 2.1 & 6.7 \\
9 & 6 & 2.0 & 0.4 & 3.6 \\
10 & 8 & 2.7 & 0.9 & 4.6 \\
ND & 4 & 1.4 & 0.0 & 2.7 \\
Total & 296 & & & \\
\hline Abbrevationyyy & & & &
\end{tabular}

Abbreviations: SLIT, sublingual immunotherapy; $\mathrm{Cl}$, confidence interval; ND, not defined.

reduction of clinical efficacy and finally, discontinuation of treatment. A treatment duration of 3-5 years has been shown to be optimal for modifying the natural history of both house dust mite ${ }^{14,15}$ and pollen ${ }^{16}$ allergy. Patients embarking on SLIT should therefore aim for this treatment duration.

The present study investigated how specialists perceive SLIT adherence issues using a questionnaire canvassing factors that are commonly believed to affect compliance. As expected, the patients' perceived clinical efficacy was the most relevant issue, and was even more important than the presence of side effects. It is worthy of note that perceived clinical efficacy may also have a negative effect, because the patient might feel that once the symptoms are controlled the treatment is no longer needed, which leads to premature cessation of treatment. ${ }^{16}$ This misconception could be addressed by patient education, as recommended in

Table 3 Ranking of cost reimbursement in adherence to SLIT

\begin{tabular}{lllll}
\hline Ranking & $\mathbf{n}$ & $\%$ & \multicolumn{2}{l}{$\mathbf{C l}$ 95\% } \\
\cline { 3 - 5 } & & & Lower & Upper \\
\hline 1 & 102 & 34.5 & 29.0 & 39.9 \\
2 & 32 & 10.8 & 7.3 & 14.3 \\
3 & 37 & 12.5 & 8.7 & 16.3 \\
4 & 26 & 8.8 & 5.6 & 12.0 \\
5 & 19 & 6.4 & 3.6 & 9.2 \\
6 & 12 & 4.1 & 1.8 & 6.3 \\
7 & 16 & 5.4 & 2.8 & 8.0 \\
8 & 20 & 6.8 & 3.9 & 9.6 \\
9 & 11 & 3.7 & 1.6 & 5.9 \\
10 & 16 & 5.4 & 2.8 & 8.0 \\
ND & 5 & 1.7 & 0.2 & 3.2 \\
Total & 296 & & & \\
\hline
\end{tabular}

Abbreviations: SLIT, sublingual immunotherapy; $\mathrm{Cl}$, confidence interval; ND, not defined.
Table 4 Ranking of treatment tolerability in adherence to SLIT

\begin{tabular}{lllll}
\hline Ranking & $\mathbf{n}$ & $\%$ & \multicolumn{2}{l}{$\mathbf{9 5 \% \mathbf { C l }}$} \\
\cline { 3 - 5 } & & & Lower & Upper \\
\hline 1 & 63 & 21.3 & 16.6 & 25.9 \\
2 & 82 & 27.7 & 22.6 & 32.8 \\
3 & 51 & 17.2 & 12.9 & 21.5 \\
4 & 19 & 6.4 & 3.6 & 9.2 \\
5 & 19 & 6.4 & 3.6 & 9.2 \\
6 & 15 & 5.1 & 2.6 & 7.6 \\
7 & 11 & 3.7 & 1.6 & 5.9 \\
8 & 15 & 5.1 & 2.6 & 7.6 \\
9 & 12 & 4.1 & 1.8 & 6.3 \\
10 & 6 & 2.0 & 0.4 & 3.6 \\
ND & 3 & 1.0 & -0.1 & 2.2 \\
Total & 296 & & & \\
\hline
\end{tabular}

Abbreviations: SLIT, sublingual immunotherapy; $\mathrm{Cl}$, confidence interval; ND, not defined.

consensus documents for any level of severity of allergy. ${ }^{17}$ By contrast, perhaps surprisingly, patient education (and parental education, when SLIT is administered children) and ease of administration were not judged by the allergists to be relevant issues. An accepted definition of patient education is "the process by which health professionals and others impart information to patients that will alter their health behaviours or improve their health status". ${ }^{18}$ It was recently demonstrated that patients receiving an educational programme on SLIT showed better compliance than patients receiving standard verbal instructions. ${ }^{19}$

Because adherence is considered to be a dynamic process, patient perception of treatment efficacy should be regularly evaluated by the attending allergist. Moreover, before beginning SLIT, physicians should give correct instructions and explanations to patients, especially concerning the treatment duration needed before assessing symptomatic relief, to avoid unrealistic expectations. The other two issues judged by the allergists to be important in compliance were tolerability and treatment cost. It is known that SLIT has a good safety profile, with local reactions in the mouth or gastrointestinal tract being the most common side effects, ${ }^{20}$ but generally these reactions are quite easy to manage and do not require

Table 5 Three-year SLIT adherence as perceived by allergists

\begin{tabular}{lllll}
\hline Adherence & $\mathbf{N}$ & $\%$ & \multicolumn{2}{l}{$\mathbf{9 5 \% \mathbf { C l }}$} \\
\cline { 3 - 5 } & & & Lower & Upper \\
\hline$>80 \%$ & 87 & 29.4 & 24.2 & 34.6 \\
$80 \%-50 \%$ & 171 & 57.8 & 49.3 & 66.3 \\
$<50 \%$ & 21 & 7.1 & 1.2 & 13.0 \\
NA & 17 & 5.7 & 0.0 & 12.5 \\
Total & 296 & & & \\
\hline
\end{tabular}

Abbreviations: SLIT, sublingual immunotherapy; $\mathrm{Cl}$, confidence interval; NA, not available. 
interruption of treatment. ${ }^{9}$ In this regard, patient education is likely to be a crucial factor in adherence.

It has been clearly demonstrated that SLIT is costeffective in terms of reducing the use of symptomatic drugs, and becomes even more cost-effective when SLIT can be stopped after an adequate treatment duration and continues to be effective in controlling allergic symptoms. ${ }^{21}$ However, it is understandable that patients absorbing the cost of SLIT may not be convinced on this issue, even after adequate information is given.

In conclusion, the results of this survey suggest that there is still room for improving the extent of patient adherence with SLIT, and that patient understanding of the specialists' perception, beliefs, and attitudes might be a fruitful advance. In particular, the perception of some aspects of treatment appears to some extent to be unrelated to the current scientific knowledge about SLIT, and may be improved by more complete information being provided. Moreover, the allergists' preference for telephone contact is another factor potentially able to improve adherence to treatment, as has been demonstrated in diabetic patients. ${ }^{22}$

\section{Acknowledgments}

The Italian Study Group on SLIT compliance: Gennaro D’Avino, Rosa Comi, Mario Lo Schiavo, Francesco Pezzuto, Carmen Montera, Antonio Pio, Maria Teresa Ielpo, Francesco Cellini, Licia Vicentini, Roberto Pecorari, Tiziana Aresu, Lucetta Capra, Elena De Benedictis, Claudio Bombi, Daniela Zauli, Annarita Vanzi, Carlo Alberto Paltrinieri, Alfeo Bondioli, Isabella Paletta, Domenica Ventura, Francesco Mei, Franco Paolini, Caterina Colangelo, Enrico Cavallucci, Francesco Cucinelli, Rosanna Tinari, Giuseppe Ermini, Valerio Beltrami, Elio Novembre, Cinzia Begliomini, Erminia Marchese, Enrico Solito, Valentino Ammannati, Gaetanino Molino, Elena Galli, Mauro Baldassini, Loreta Di Michele, Mauro Calvani, Mirella Gidaro, Alberto Venuti, Enrico Li Bianchi, Fulvio Benassi, Donatella Pocobelli, Paola Zangari, Maria Grazia De Rocco, Antonella Lo Vecchio, Giuseppe Pingitore, Orazio Grimaldi, Domenico Schiavino, Nicola Perrone, Maria Antonietta Frieri, Valerio Di Rienzo, Salvatore Tripodi, Alessandro Scarpa, Mirala Tomsic, Roberta Bonaguro, Gian Enrico Senna, Alberto Sirena, Franca Turatello, Stefano Crescioli, Elisabetta Favero, Lucia Billeri, Fulvia Chieco Bianchi, Carla Gemignani, Mario Zanforlin, Maria Angiola Crivellaro, Birgir Hendrick, Adriana Maltauro, Simonetta Masieri, Maria Elisabetta Conte, Mario Fama, Marcella Pozzan, Patrizia Bonadonna, Stefania Casanova, Elisabetta Vallerani, Michele
Schiappoli, Franco Borghesan, Giuseppina Giro, Stefano Casotto, Loredana Berardino, Giovanna Zanoni, Renato Ariano, Riccardo Aquilina, Renato Pellegrino, Pietro Marsico, Angelica Del Giudice, Giuseppe Narzisi, Valerio Tomaselli, Gianfranco Fornaca, Medicina, Mauro Favro, Bruna Loperfido, Cesare Gallo, Sebastiano Buffoni, Federica Gani, Paolo Raviolo, Stefano Faggionato, Tiziana Truffelli, Livio Vivalda, Monica Albano, Renato Enzo Rossi, Giovanni Lattuada, Fausto Bona, Luisella Quaglio, Adriana Chiesa, Marco Trapani, Rosalba Seminara, Beniamino Cucchi, Silvana Oderda, Giovanni Borio, Giuseppe Galeasso, Paolo Garbaccio, Antonino De Marco, Fulvia Marengo, Gianni Cadario, Silvia Manzoni, Claudio Vinay, Antonio Curcio, Alberto Silvestri, Antonella Peduto, Gian Galeazzo RiarioSforza, Anna Maria Forgnone, Piercarlo Barocelli, Nicoletta Tartaglia, Gianfranco Feyles, Adriana Giacone, Vittorio Ricca, Giuseppe Guida, Franco Nebiolo, Luisa Bommarito, Enrico Heffler, Felice Vietti, Maurizio Galimberti, Eleonora Savi, Antonio Pappacoda, Paolo Bottero, Stefania Porcu, Giuseppe Felice, Daniele Berra, Maria Francesca Spina, Valerio Pravettoni, Ambra M Calamari, Elena Varin, Enrico Iemoli, Daniele Lietti, Daniele Ghiglioni, Alessandro Fiocchi, Anna Tosi, Mariangela Poppa, Antonio Caviglia, Massimo Restuccia, Marina Russello, Paolo Alciato, Giuseppina Manzotti, Emanuela Ranghino, Giorgio Luraschi, Andrea Rapetti, Federica Rivolta, Flavio Allegri, Luigi Terracciano, Fabio Agostinis, Pier Paolo Piras, Giuseppe Ronchi, Giulio Gaspardini, Vincenzo Caria, Fortunato Tolu, Dina Fantasia, Paola Carta, Anna Moraschini, Roberto Quilleri, Antonietta Santelli, Paolo Prandini, Giorgia Del Giudice, Aldo Apollonio, Lucio Bonazza, Maria Teresa Franzini, Silvia Branchi, Mario Zanca, Sandro Rinaldi, Luca Catelli, Tullio Zanoletti, Cristina Cosentino, Fabrizio Della Torre, Luigi Cremonte, Dario Musazzi, Claudia Suli, Loredana Rivolta, Anna Ottolenghi, Gianluca Marino, Giuseppe Sterza, Renato Sambugaro, Antonella Orlandini, Paola Minale, Susanna Voltolini, Donatella Bignardi, Pinuccia Omodeo, Antonio Tiri, Stefania Milani, Barbara Ronchi, Giovanna Licardi, Paola Bruni, Joseph Scibilia, Jan Schroeder, Francesca Crosti, Angela Maltagliati, Maria R Alesina, Marisa Mosca, Gennaro Leone, Giorgio Napolitano, Giuseppe Di Gruttola, Guglielmo Scala, Silvia Mascio, Annamaria Valente, Ilaria Marchetiello, Romano Catello, Antonio Gazulli, Antonio Del Prete, Alfonso M Varricchio, Agostino Carbone, Angelo Forestieri, Maria Stillitano, Luigi Leonetti, Enrico Tirroni, Francesco Castellano, Francesco Abbagnara, Francesco Romano, Carmelo Levanti, Marcello Cilia, Rocco Longo, Angelo Ferrari, Alberto 
Ferrari, Roberto Merenda, Antonio Di Ponti, Enrico Guercio, Lorenzo Surace, Giuseppe Ammendola, Francesco Tansella, Lucia Peccarisi, Lorenza Stragapede, Michele Minenna, Massimo Granato, Nicola Fuiano, Alessandro Pannofino, Simona Ciuffreda, Angela Giannotta, Giuseppe Morero, Lucia D’Oronzio, Giordano Taddeo, Eustachio Nettis, Giuseppe Cinquepalmi, Carlo Lamanna, Fulvio Mastrandrea, Mauro Minelli, Francesco Salamino, Lionello Muratore, Francesco Latorre, Carlo Quarta, Maria Ventura, Giannamaria D’Ippolito, Francesco Giannoccaro, Porzia Dambra, Luigi Pinto, Michele Triggiani, Giorgio Munno, Giacomo Manfredi, Giuseppe Lonero, Vincenzo Damiano, Giuseppe Errico, Elisabetta Di Leo, Francesco Manzari, Virgilio Spagna, Augusto Arsieni, Aurelia Matarrese, Giuseppe Mazzarella, Giovanni Scarcia, Rosa Scarano, Antonio Ferrannini, Antonio Pastore, Patrizia Maionchi, Luigi Filannino, Margherita Tria, Giampiero Giuliano, Elisabetta Damiani, Nicola Scichilone, Margherita Marchese, Anna Lucania, Mauro Marino, Luigi Strazzeri, Silvana Tumminello, Giovanni I Vitale, Salvatore Gulotta, Giuseppe Gragotto, Marcello Zambito, Domenico Greco, Giuseppe Valenti, Giorgio Licitra, Enzo Cannata, Rosalia Filpi, Mariarita Contraffatto, Stefania Sichili, Salvatore Randazzo, Giovanna Scarantino, Bruno Lo Porto, Franco Pavone, Claudio Di Bartolo, Antonino Paternò, Francesca Rapisarda, Erasmo Laudani, Salvatore Leonardi, Vincenzo Padua, Gaetano Cabibbo, Giuseppe Marino Guzzardi, Francesco Deluca, Claudio Agozzino, Rosa Pettinato, Marco Ghini.

\section{Disclosure}

The authors report no conflict of interest in this work.

\section{References}

1. Bousquet J, van Cauwenberge P. Allergic rhinitis and its impact on asthma. J Allergy Clin Immunol. 2001;108(Suppl 5):147-334.

2. Compalati E, Penagos M, Tarantini F, et al. Specific immunotherapy for respiratory allergy: State of the art according to current meta-analyses. Ann Allergy Asthma Immunol. 2009;102:22-28.

3. Gidaro GB, Frati F, Sensi L, et al. The safety of sublingual-swallow immunotherapy: An analysis of published studies. Clin Exp Allergy. 2005;35:565-571.

4. Incorvaia C, Mauro M, Ridolo E, et al. Patient's compliance with allergen immunotherapy. Patient Prefer Adherence. 2008;2:247-250.
5. Haynes RB, Sackett DL, Taylor DW. Compliance in Healthcare Baltimore, MD: Johns Hopkins University Press; 1979.

6. Pajno GB, Vita D, Caminiti L, et al. Children's compliance with allergen immunotherapy according to administration routes. J Allergy Clin Immunol. 2005;116:1380-1381.

7. Passalacqua G, Musarra A, Pecora S, et al. Quantitative assessment of the compliance with a once-daily sublingual immunotherapy regimen in real life (EASY Project: Evaluation of a novel SLIT formulation during a year). J Allergy Clin Immunol. 2006;117:946-948.

8. Passalacqua G, Musarra A, Pecora S, et al. Quantitative assessment of the compliance with a once-daily sublingual immunotherapy in children (EASY Project: Evaluation of a novel SLIT formulation during a year). Pediatr Allergy Immunol. 2007;18:58-62.

9. Frati F, Sensi L, Di Rienzo V, et al. A model for management of sublingual immunotherapy. Eur Ann Allergy Clin Immunol. 2003;35: $56-60$.

10. Bousquet PJ, Combescure C, Klossek JM, et al. Change in visual analogue scale score in a pragmatic randomized cluster trial of allergic rhinitis. J Allergy Clin Immunol. 2009;123:1349-1354.

11. Sabate E. Adherence to Long Term Therapies: Evidence for Action. Geneva, Switzerland: World Health Organization; 2003:47-58.

12. Osterberg L, Blashke T. Adherence to medication. $N$ Engl J Med. 2005;353:487-497.

13. Baiardini I, Braido F, Tarantini F, et al. ARIA-suggested drugs for allergic rhinitis: What impact on quality of life? A GA2LEN review. Allergy. 2008;63:660-669.

14. Di Rienzo V, Marcucci F, Puccinelli P, et al. Long-lasting effect of sublingual immunotherapy in children with asthma due to house dust mite: A 10-year prospective study. Clin Exp Allergy. 2003;33:206-210.

15. Ozdemir C, Yazi D, Gocmen I, et al. Efficacy of long-term sublingual immunotherapy as an adjunct to pharmacotherapy in house dust miteallergic children with asthma. Pediatr Allergy Immunol. 2007;18: 508-515.

16. Novembre E, Galli E, Landi F, et al. Coseasonal sublingual immunotherapy reduces the development of asthma in children with allergic rhinoconjunctivitis. J Allergy Clin Immunol. 2004;114:851-857.

17. Bousquet J, Khaltaev N, Cruz AA, et al. Allergic Rhinitis and its Impact on Asthma (ARIA) 2008 update (in collaboration with the World Health Organization, GA2LEN and AllerGen). Allergy. 2008;63 Suppl 86:8-160.

18. Koongstvedt PR. The Managed Health Care Handbook. 4th ed. Philadelphia, PA: Aspen Publishers Inc.; 2001.

19. Incorvaia C, Rapetti A, Scurati S, et al. Importance of patient's education in favouring compliance with sublingual immunotherapy. Allergy. $2010 \mathrm{Feb} 26$. [Epub ahead of print].

20. Gidaro GB, Marcucci F, Sensi L, et al. The safety of sublingual-swallow immunotherapy: An analysis of published studies. Clin Exp Allergy. 2005;35:565-571.

21. Berto P, Frati F, Incorvaia C. Economic studies of immunotherapy: A review. Curr Opin Allergy Clin Immunol. 2008;8:585-589.

22. Brekke HK, Sunesson A, Lenner RA. Unannounced telephone interviews: A useful and positively received tool in the reinforcement of lifesyle intervention. Patient Prefer Adherence. 2009;3:355-362.
Patient Preference and Adherence

\section{Publish your work in this journal}

Patient Preference and Adherence is an international, peer-reviewed, open access journal that focusing on the growing importance of patient preference and adherence throughout the therapeutic continuum. Patient satisfaction, acceptability, quality of life, compliance, persistence and their role in developing new therapeutic modalities and compounds to

\section{Dovepress}

optimize clinical outcomes for existing disease states are major areas of interest. This journal has been accepted for indexing on PubMed Central. The manuscript management system is completely online and includes a very quick and fair peer-review system. Visit http://www.dovepress.com/ testimonials.php to read real quotes from published authors. 